\title{
Patterns of Diabetes Screening and Prediabetes Treatment during Office Visits in the US
}

\author{
Kayce M. Shealy, PharmD, BCPS, BCACP, CDE, Jun Wu, PhD, \\ Jessica Waites, PharmD Candidate, Nancy A. Taylor, RPh, CDE, \\ and G. Blair Sarbacker, PharmD, BCACP
}

Introduction: The American Diabetes Association recommends annual screenings for prediabetes if the patient meets the suggested requirements. The overall prevalence of prediabetes has decreased from an estimated 86 million adults in 2012 to 84.1 million adults in 2015 in the United States. Along with lifestyle modifications, the use of metformin as a treatment option or in combination has shown a decrease in weight and health care costs. This study was designed to review the prevalence of screening and treatment of prediabetes in the United States by using the National Ambulatory Medical Care Survey, as well as identify any factors associated with screenings and treatment.

Methods: The National Ambulatory Medical Care Survey was used to examine a study sample of office visits between 2012 and 2015, reviewing the prevalence of screenings and lab services ordered or provided at each patient visit. Inclusion criteria consisted of the recommendations given by the American Diabetes Association including any patient $\geq 45$ years or adult patient $<45$ years with a body mass index of $\geq 25 \mathrm{~kg} / \mathrm{m}^{2}$ and an additional risk factor. Patients with a previous diagnosis of diabetes were excluded from the sample.

Results: A total of 105,721 office visits (2012 to 2015) were included in the analysis. The diabetes screening prevalence increased from $10 \%$ in 2012 to $13.4 \%$ in 2015 . Metformin $(n=140,76.1 \%)$ was the most common antidiabetic medication prescribed to treat prediabetes.

Conclusions: The prevalence of diabetes screening during office visits remained lower than $15 \%$ between 2012 and 2015 in the United States. Physicians primarily prescribe lifestyle modifications, including a healthy diet and exercise, with metformin being used in some cases for the prevention of diabetes. (J Am Board Fam Med 2019;32:209-217.)

Keywords: Diabetes Mellitus, Health Care Surveys, Hypoglycemic Drugs, Metformin, Prediabetes, Prevalence

The American Diabetes Association (ADA) recommends that asymptomatic patients who are overweight (body mass index $[\mathrm{BMI}] \geq 25 \mathrm{~kg} / \mathrm{m}^{2}$ ) with an additional risk factor, such as the presence of cardiovascular disease (CVD), hypertension, polycystic ovarian syndrome (PCOS), or high risk race or ethnic group (African American, Native American,

This article was externally peer reviewed.

Submitted 3 September 2018; revised 6 December 2018; accepted 9 December 2018.

From Presbyterian College School of Pharmacy, Clinton, SC (KMS, JW, JW, NAT, GBS).

Funding: none.

Conflict of interest: none declared.

Corresponding author: Kayce M. Shealy, PharmD, BCPS, BCACP, CDE, Pharmacy Practice, Presbyterian College School of Pharmacy, 307 N. Broad St., Clinton, SC 29325 (E-mail: kmshealy@presby.edu).
Latino, Asian American, or Pacific Islander), as well as all adults aged at least 45 years be screened for the presence of type 2 diabetes mellitus (DM) every 1 to 3 years depending on screening results. ${ }^{1}$ Similarly, the American Association of Clinical Endocrinologists (AACE) and American College of Endocrinology recommend screening in asymptomatic adults who are overweight with additional risk factors, as well as adults at least 45 years old without risk factors. ${ }^{2}$ Both the American Academy of Family Physicians and the United States Preventive Services Task Force (USPSTF) recommend screening individuals aged 40 to 70 years who are overweight or obese, and the USPSTF recommends earlier screening if additional risk factors are present, such as family history of diabetes, certain ethnic or racial groups, or personal history of gestational diabetes 
or PCOS. ${ }^{3,4}$ Patients with abnormal results that are not indicative of overt DM are classified with prediabetes by the ADA and AACE and should be screened for DM annually. ${ }^{1,2}$ Those with normal screening results may be tested less frequently, such as every 3 years. ${ }^{1-4}$

The overall prevalence of prediabetes has decreased from an estimated 86 million adults in 2012 to 84.1 million adults in 2015 in the United States based on fasting glucose or hemoglobin A1C results. ${ }^{5,6}$ However, the prevalence of prediabetes in the geriatric population (at least 65 years of age) is higher than other age groups, estimated at approximately 1 in 2 older adults in $2015 .^{7}$

Patients with prediabetes have an increased risk of developing DM in the future. It is estimated that $5 \%$ to $10 \%$ of patients with prediabetes develop overt diabetes annually and almost $75 \%$ of patients with prediabetes will progress to overt diabetes in their lifetime. ${ }^{8,9}$ Approximately $12 \%$ of the US adult population was estimated to have DM in 2015 , although only $9.3 \%$ of the adult population was officially diagnosed. ${ }^{7}$ More than $25 \%$ of the US population at least 65 years of age are estimated to have DM, including those who are undiagnosed. There is a higher prevalence in American Indians, Alaska Natives, non-Hispanic African Americans, and Hispanic people compared with non-Hispanic Caucasians. Although the overall prevalence of DM has increased, the incidence of DM has actually decreased compared with $2014 .{ }^{6}$ However, the cost of DM continues to rise. Treatment of DM was estimated to cost $\$ 237$ billion in direct medical costs and approximately $\$ 90$ billion due to lost productivity in 2017. ${ }^{10}$ On average, patients with DM incurred over 2 times more medical expenses compared with those without DM, with approximately $\$ 9,600$ directly attributed to DM in 2017.

More attention has been placed to preventing $\mathrm{DM}$ in recent years, including the launch of the National Diabetes Prevention Program (DPP) by the Centers for Disease Control and Prevention (CDC) in 2010, and a prediabetes awareness campaign by the CDC, ADA, American Medical Association, and the Ad Council in 2016 entitled Prevent Diabetes STAT (Screen, Test, Act Today). ${ }^{6,11}$ The awareness campaign encourages people to determine their risk of developing prediabetes and to engage in interventions to prevent or delay DM. The campaign also provides a toolkit for health care professionals to help engage patients and the health care team and also incorporate screening and referral to lifestyle programs into practice. Similarly, the ADA guidelines encourage interventions, such as intensive lifestyle interventions or certain pharmacologic therapies such as metformin. ${ }^{12}$ Lifestyle interventions, including $5 \%$ to $7 \%$ weight loss and 150 minutes of weekly physical activity, have been shown to reduce the number of diagnoses of DM in the short-term (58\% over 3 years) and the longterm (34\% at 10 years). In addition, metformin may be as effective as lifestyle interventions in extremely obese patients. ${ }^{6,12}$

Identifying patients at risk for developing DM and using effective interventions to prevent DM in those at risk may lead to reduced health care spending by avoiding the costs associated with treating overt DM mentioned previously. It is likely that many US adults are not being screened and may be missing opportunities to delay or prevent the progression to DM given the high estimates of the proportion of the population who are unaware of either a diagnosis of DM or prediabetes. There is also a lack of complete agreement on who qualifies for screening, as evidenced by the differences in guidelines from national organizations. ${ }^{1-4}$ This study sought to identify the prevalence of DM screening according to diabetes-specific organization guidelines and any associated factors with screenings by using a national database of ambulatory care visits and to identify treatment recommendations for those patients identified as having prediabetes.

\section{Methods}

\section{Data Source}

The National Ambulatory Medical Care Survey (NAMCS) is a national sample survey of officebased visits conducted annually by the National Center for Health Statistics, a component of the National Health Care Survey managed by the CDC in the Unitd States. ${ }^{13}$ NAMCS uses a 2-stage probability sampling design, with physicians selected in the first stage and reporting weeks assigned for visits in the second stage. The findings based on NAMCS represent a national sample of visits to non-federally-employed office-based physicians involved in direct patient care. NAMCS collected event-level information for each office visit, including patient demographic and clinical characteristics, diagnosis of disease, reasons for vis- 
its, examinations or procedures provided during visits, medications prescribed, physician specialties, and practice site information. The study was approved by the Institutional Review Board of Presbyterian College.

\section{Study Sample}

The study sample was yielded by combining NAMCS data (2012 to 2015) and applying the following inclusion and exclusion criteria based on ADA Standards of Medical Care for testing for diabetes or prediabetes in asymptomatic adults. ${ }^{1}$ This study included all visits involving patients $\geq 45$ years and visits involving patients 18 to 44 years and $\mathrm{BMI} \geq 25$ with 1 of the following risk factors: African American, Hispanic, Asian, Pacific Island, Native American, hypertension, CVD, PCOS, or abnormal high density lipoprotein (HDL, $\leq 35 \mathrm{mg} / \mathrm{dL}$ ) or triglyceride (TG, $\geq 250 \mathrm{mg} / \mathrm{dL}$ ) levels. Visits involving patients with diabetes were excluded from the study sample. CVD included congestive heart failure (CHF), coronary artery disease (CAD), ischemic heart disease, and history of myocardial infarction (MI). Two groups were created for analysis: (1) visits with provision of screening for diabetes and (2) visits without provision of screening for diabetes.

NACMS provided 3 sources to identify CVD, hypertension, PCOS, and diabetes. These include the following: (1) NAMCS measured multiple chronic conditions selected from the Office of the Assistant Secretary for Health's List of Selected Chronic Conditions. ${ }^{14}$ Physicians reported hypertension, $\mathrm{CHF}$, and $\mathrm{CAD}$ via checkbox provided in the survey along with the question "Regardless of the diagnoses written above, does the patient now have (the following diseases)?" The variable CAD also included ischemic heart disease and history of MI. (2) The provider's diagnosis for the visit as reported by International Classification of Diseases, Ninth Revision (ICD-9) codes (up to 5 diagnoses) for each visit. Office of the Assistant Secretary for Health provided corresponding ICD-9 codes of chronic conditions in NAMCS. ${ }^{14}$ PCOS was identified by ICD-9 codes (256.4). (3) The patient's reasons for the visit (up to 5 reasons); NAMCS collected information on the patient's reason for the visit and coded according to A Reason for Visit Classification for Ambulatory Care. All visits reporting reasons associated with the conditions mentioned in the inclusion and exclusion criteria were identified by using Reason for Visit
Classification codes. In addition, HDL and TG tests represented the most recent results during the 12 months before the visits.

\section{Outcome Measures}

The primary outcome was provision of screening for diabetes during office visits. NAMCS measured a variety of services ordered or provided at the visits, including examinations, lab tests, imaging, procedures, treatments, and health education/counseling. The 3 variables (lipid profile, fasting glucose, and A1C tests) were selected to reflect screening for diabetes. The presence of either fasting glucose or A1C provided during the visits was defined as provision of screening for diabetes. Lipid profile was captured, as abnormal levels of HDL or TG are considered risk factors for developing DM.

The secondary outcomes were prevalence of prediabetes in the study sample and treatment patterns for prediabetes. Prediabetes was identified by elevations in recent fasting blood glucose (100 to $126 \mathrm{mg} / \mathrm{dL})$ or $\mathrm{A} 1 \mathrm{C}(5.7 \%$ to $6.4 \%)$, or by the ICD-9 codes (790.21, 790.22, or 790.29), reflecting impaired fasting glucose, impaired glucose tolerance, or other abnormal glucose levels, respectively. ${ }^{1}$ Visits with prediabetes were defined if any 1 of the above 3 criteria was met. Treatments for prediabetes included lifestyle management and antihyperglycemic drugs. ${ }^{12}$ NAMCS measured health education/counseling that was provided or ordered at the visits and referrals for the conditions. The 3 variables diet/nutrition, exercise, and weight management were used to identify lifestyle management provision during the prediabetes-related visits. Diet/nutrition education contained topics related to the foods and beverages consumed by the patient and instructions to prevent diabetes. Exercise education included the topics related to the patient's physical conditioning or fitness, such as health promotion and disease prevention. Weight management education refers to information provided to the patient to assist in the goal of weight reduction. The antihyperglycemic medications prescribed during prediabetes-related visits were identified by using the 3-level Multum classification of therapeutic classes developed by Lexicon Plus database. ${ }^{15}$ The first level code identified metabolic agents and then the second level code identified antihyperglycemic agents. The third level codes identified various classes of antihyperglycemic medications. Based on the distribution of antihyperglycemic medica- 
tions prescribed during prediabetes-related visits in our study sample, the antihyperglycemic medications were collapsed into biguanide, sulfonylureas, thiazolidinediones, and others (incretin mimetics, sodium-glucose cotransporter 2, and combination therapy). Any 1 of the above antihyperglycemic medications prescribed was defined as provision of antihyperglycemic medication treatment for prediabetes.

\section{Data Analysis}

Frequencies and relative frequencies (\%) described the characteristics of the study sample, trend and patterns of screening for diabetes (2012 to 2015), and patterns of treatment for prediabetes. $\chi^{2}$ tests compared characteristics of the sample visits between screening provided during visits and no screening. All candidate variables associated with diabetes screening were included in a multivariate logistic regression model. The potential factors included in the logistic regression model were age, race, sex, BMI, primary payer, smoking, hypertension, $\mathrm{CHF}$, CAD, abnormal lipids, prediabetes, total number of chronic conditions, physician specialty, office location, and geographic region. The statistical significance was set at $P<.05$. NAMCS uses multistage probability sampling, and the data contains patient visit weights for all the visits. In the analysis, the patient weights were aggregated by using SAS survey procedures to produce national estimates from the study sample. All statistical analyses were performed by using SAS 9.4.

\section{Results}

A total of 105,721 office visits meeting criteria were identified from NAMCS data (2012 to 2015), representing 1.9 billion visits in the United States from 2012 to 2015 (Figure 1). Of these, $94.5 \%$ of the visits involved patients $\geq 45$ years and $5.5 \%$ of the visits involved patients $<45$ years with at least 1 risk factor for future DM (Figure 1). Screening for diabetes was provided at $11.9 \%(n=8375)$ of the visits included in the study sample.

As shown in Table 1, the prevalence of prediabetes in the study sample was $6 \%$. The diabetes screening group had a higher prevalence of prediabetes $(16.7 \%)$ than the no screening group (4.6\%, $P<.001)$. Based on multivariate logistic regression, nonwhite race, higher BMI, private insurance, abnormal lipid level, prediabetes, greater number
Figure 1. Study sample of eligible visits with risk factors for prediabetes, NAMCS 2012-2015. CVD, cardiovascular disease; HTN, hypertension; PCOS, polycystic ovarian syndrome.

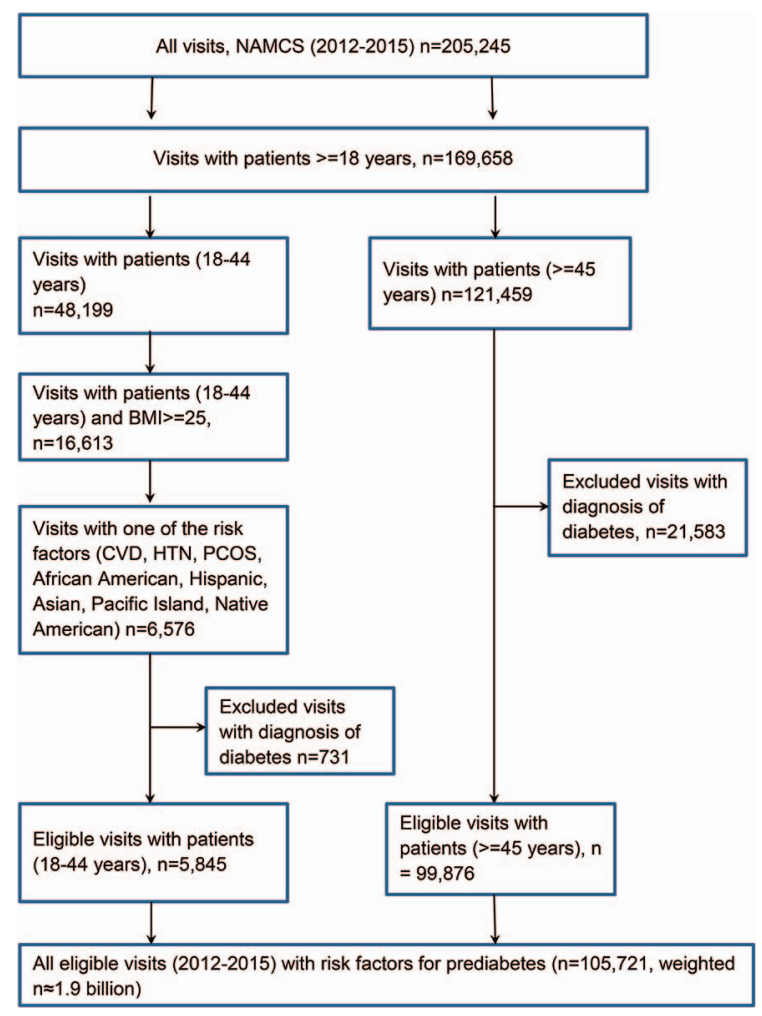

of chronic conditions, seeing primary care providers, and living in northeast increased the likelihood of screening provision during visits. Visits involving black and other race patients were 28\% (odds ratio [OR],1.28; 95\% CI, 1.03-1.58) and 44\% (OR, 1.44; $95 \%$ CI, $1.14-1.82$ ) more likely to provide screening than the visits involving white patients. Compared with patients with a BMI $<18.5$, screening was 3.17 times (OR, 3.17; 95\% CI, 2.63-3.81) and 2.08 times (OR, 2.08; 95\% CI, 1.72-2.51) more likely to be provided during visits when patients were overweight and obese, respectively. Visits involving patients covered by public insurance were $21 \%$ (OR, 0.79; 95\% CI, 0.69-0.91) less likely to provide screening than those involving patients covered by private insurance. Compared with seeing primary care providers, seeing CVD specialists reduced the screening provision by $62 \%$ (OR, 0.38; 95\% CI, 0.25-0.58). Screening was 1.69 times (OR, 1.69; 95\% CI, 1.30-2.20) and 2.56 times (OR, 2.56; 95\% CI, 2.19-3.00) more likely to be provided during visits to patients with abnormal lipid levels and prediabetes than those not. Com- 
Table 1. Characteristics of Visit Sample and Factors Associated with Diabetes Screening $(\mathrm{n}=\mathbf{1 0 5 , 7 2 1})$

\begin{tabular}{|c|c|c|c|c|}
\hline Variable & $\begin{array}{c}\text { Total No. } \\
(\mathrm{n}=105,721)\end{array}$ & $\begin{array}{l}\text { Diabetes Screening } \\
(\mathrm{n}=8,375), \mathrm{N} \\
(\text { weighted \%) }\end{array}$ & $\begin{array}{c}\text { No Diabetes Screening } \\
(\mathrm{n}=97,346), \mathrm{N} \\
(\text { weighted } \%)\end{array}$ & $\begin{array}{l}\text { Adjusted OR of Screening } \\
\qquad(95 \% \mathrm{CI})\end{array}$ \\
\hline \multicolumn{5}{|l|}{ Age (years) } \\
\hline 18 to 44 & $5,845(6.8)$ & $653(8.6)$ & $5,192(6.6)$ & 1.00 \\
\hline 45 to 64 & $52,100(49.7)$ & $4,302(53.6)$ & $47,798(49.2)$ & $1.18(0.96-1.46)$ \\
\hline$\geq 65$ & $47,776(43.5)$ & $3,420(37.8)$ & $44,356(44.2)$ & $0.86(0.69-1.06)$ \\
\hline \multicolumn{5}{|l|}{ Race } \\
\hline White & $92,347(83.8)$ & $7,043(78.2)$ & $85,304(84.5)$ & 1.00 \\
\hline Black & $9,263(10.5)$ & $918(14.9)$ & $8,345(9.9)$ & $1.28(1.03-1.58)$ \\
\hline Other $^{*}$ & $4,111(5.7)$ & $414(7.0)$ & $3,697(5.5)$ & $1.44(1.14-1.82)$ \\
\hline \multicolumn{5}{|l|}{ Sex } \\
\hline Female & $60,831(58.5)$ & $4,633(56.9)$ & $56,198(58.7)$ & 1.00 \\
\hline Male & $44,890(41.5)$ & $3,742(43.1)$ & $41,148(41.3)$ & $0.99(0.88-1.11)$ \\
\hline \multicolumn{5}{|l|}{ BMI } \\
\hline$<18.5$ & $42,799(36.1)$ & $1,451(13.1)$ & $41,348(39.1)$ & 1.00 \\
\hline 18.5 to 25 & $16,062(16.4)$ & $1,536(19.8)$ & $14,526(15.9)$ & $2.63(2.15-3.21)$ \\
\hline 25 to 30 & $22,692(23.6)$ & $2,703(36.3)$ & $19,989(21.9)$ & $3.17(2.63-3.81)$ \\
\hline$\geq 30$ & $24,168(24.0)$ & $2,685(30.7)$ & $21,483(23.1)$ & $2.08(1.72-2.51)$ \\
\hline \multicolumn{5}{|l|}{ Primary payer } \\
\hline Private & $46,101(44.3)$ & $4,085(50.5)$ & $42,016(43.4)$ & 1.00 \\
\hline Medicare/Medicaid & $46,584(43.4)$ & $3,431(40.3)$ & $43,153(43.8)$ & $0.79(0.69-0.91)$ \\
\hline Uninsured & $4,006(4.6)$ & $164(1.9)$ & $3,842(5.0)$ & $0.52(0.37-0.73)$ \\
\hline Other $^{\dagger}$ & $9,030(7.7)$ & $695(7.4)$ & $8,335(7.8)$ & $0.87(0.61-1.25)$ \\
\hline Smoking & $13,764(12.8)$ & $1,333(15.7)$ & $12,431(12.5)$ & $0.98(0.87-1.10)$ \\
\hline \multicolumn{5}{|l|}{ Comorbidity } \\
\hline Hypertension & $36,729(37.1)$ & $4,512(53.0)$ & $32,217(35.0)$ & $1.05(0.92-1.20)$ \\
\hline $\mathrm{CHF}$ & $3,365(3.3)$ & $365(3.4)$ & $3,000(3.3)$ & $0.75(0.58-0.96)$ \\
\hline CAD & $4,996(5.4)$ & $740(7.7)$ & $4,256(5.1)$ & $0.97(0.79-1.19)$ \\
\hline PCOS & $70(0.08)$ & $19(0.2)$ & $51(0.1)$ & $\ldots$ \\
\hline Abnormal lipids $\$$ & $1,323(1.3)$ & $362(3.2)$ & $961(1.1)$ & $1.69(1.30-2.20)$ \\
\hline Prediabetes & $5,406(6.0)$ & $1,523(16.7)$ & $3,883(4.6)$ & $2.56(2.19-3.00)$ \\
\hline \multicolumn{5}{|c|}{ Total no. of chronic conditions } \\
\hline 0 & $34,527(30.5)$ & $1,241(14.8)$ & $33,286(32.6)$ & 1.00 \\
\hline 1 & $32,263(30.2)$ & $2,102(25.6)$ & $30,161(30.8)$ & $1.56(1.33-1.83)$ \\
\hline 2 & $20,470(20.0)$ & $2,362(26.6)$ & $18,108(19.0)$ & $2.26(1.87-2.74)$ \\
\hline$>2$ & $18,461(19.4)$ & $2,670(33.0)$ & $15,791(17.6)$ & $2.97(2.37-3.72)$ \\
\hline \multicolumn{5}{|l|}{ Physician Specialty } \\
\hline Family/Internal medicine & $18,378(29.3)$ & $3,959(62.8)$ & $14,419(24.8)$ & 1.00 \\
\hline CVD specialty & $3,730(4.6)$ & $520(5.0)$ & $3,210(4.5)$ & $0.38(0.25-0.58)$ \\
\hline Other & $83,613(66.0)$ & $3,896(32.2)$ & $79,717(70.7)$ & $0.26(0.22-0.31)$ \\
\hline \multicolumn{5}{|l|}{ Physician Office } \\
\hline Metropolitan area & $93,411(91.7)$ & $7,350(92.3)$ & 86,061 (91.6) & $0.83(0.67-1.03)$ \\
\hline \multicolumn{5}{|l|}{ Region } \\
\hline Northeast & $15,449(21.5)$ & $1,518(27.5)$ & $13,931(20.7)$ & 1.00 \\
\hline Midwest & $28,363(17.9)$ & 2,207 (16.9) & $26,156(18.0)$ & $0.61(0.48-0.78)$ \\
\hline South & $37,030(37.4)$ & $2,972(36.7)$ & $34,058(37.5)$ & $0.67(0.52-0.88)$ \\
\hline West & $24,879(23.2)$ & $1,678(18.9)$ & $23,201(23.8)$ & $0.52(0.40-0.67)$ \\
\hline
\end{tabular}

*Other races include Hispanic, Asian, Native American, and multiple races.

†Other sources of payments include worker's compensation, other, and unknown.

$\ddagger$ Not a reliable estimate due to a small number $(<30)$ of patients with PCOS in the screening group.

$\$$ High density lipoprotein (HDL) $<35 \mathrm{mg} / \mathrm{dL}$ or triglycerides (TG) $>250 \mathrm{mg} / \mathrm{dL}$

BMI, body mass index; CI, confidence interval; CVD, cardiovascular disease; OR, odds ratio; PCOS, polycystic ovarian syndrome;

$\mathrm{CHF}$ congestive heart failure; $\mathrm{CAD}$, coronary artery disease. 
Figure 2. Trend of diabetes screening prevalence in the study sample (2012 to 2015).

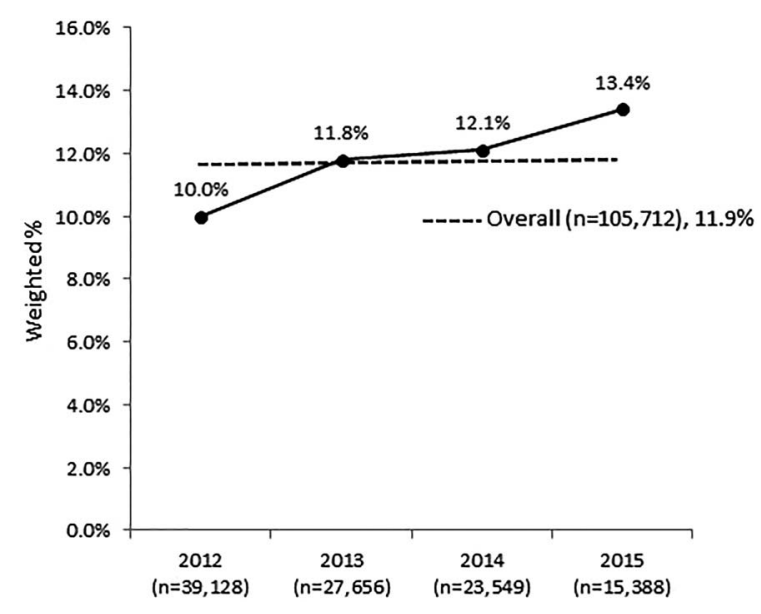

pared with no chronic conditions, when the number of chronic conditions increased from 1 to $>2$, the likelihood of screening provision increased from $56 \%(\mathrm{OR}, 1.56$; $95 \% \mathrm{CI}, 1.33-1.83)$ to $197 \%$ (OR, 2.97; 95\% CI, 2.37-3.72), respectively.

The prevalence of diabetes screening provided during visits gradually increased from 10\% in 2012 to $13.4 \%$ in 2015 , as shown in Figure 2. However, the overall prevalence of diabetes screening remained lower than $15 \%$. Among all visits with screening $(n=8375)$ in Figure 3, a lipid profile was provided or ordered at $86.6 \%$ of the visits and fasting blood glucose and A1C tests were provided or ordered at $31.6 \%$ and $33.8 \%$ of the visits, respectively.

Figure 4 presents the treatment patterns provided during visits with prediabetes. Among the visits with prediabetes $(\mathrm{n}=5,406)$, lifestyle management was provided at $21.3 \%$ of the visits and antihyperglycemic drugs were prescribed at nearly $3 \%$ of the visits. Metformin accounted for $76.1 \%$ of the prediabetes-related visits involving antihyperglycemic medication prescribed.

\section{Discussion}

More than 7 million adults are estimated to have undiagnosed diabetes, with less than 10 million US adults being aware that they have prediabetes. ${ }^{6} \mathrm{Al}-$ though it is encouraging that the prevalence of diabetes screening increased over time from $10 \%$ in 2012 to $13.4 \%$ in 2015 , the overall annual screening for DM remains low. These results are lower than previously published studies that used the $\mathrm{Na}$ - tional Health and Nutrition Examination Survey. ${ }^{16,17}$ In 1 study, only $46 \%$ of patients meeting ADA criteria for DM screening self-reported testing in the past 3 years. ${ }^{16}$ In the other study, only $37.4 \%$ of respondents eligible for DM screening based on ADA criteria were tested within the past 3 years. ${ }^{17}$ A survey of primary care physicians that included data extraction from individual electronic medical records revealed that a majority relied on the ADA guidelines for screening at least $50 \%$ of time but not all the time. ${ }^{18}$ However, a review of the electronic medical records indicated discordance with screening practices, where physicians screened less frequently than reported. Perhaps providers are unaware of how frequently (or infrequently) screening actually happens.

In this study, more patients 45 years of age or older were screened compared to those under 45 years of age, although there was no statistically significant difference. This is similar to self-reported testing previously published. ${ }^{16}$ In addition, this finding supports a consensus of the recommendations for more routine screening in older patients. ${ }^{1-4}$ Only $11 \%$ of eligible patients aged 18 to 44 years were screened in the study sample. The incidence of DM in youth increased approximately $4.8 \%$ from 2002 to 2012, with approximately 5,300 people aged 10 to 19 years diagnosed with DM in 2011 to $2012 .^{6}$ This trend identifies a need to screen for DM earlier, particularly if there are risk factors present. The ADA, AACE, and USPSTF recommend earlier screening in the presence of risk factors. ${ }^{1,2,4}$

Although the prevalence of prediabetes is highest in the older population (65 years of age and older) according to national data, fewer patients in this age category received screening. This popula-

Figure 3. Prevalence of laboratory testing provided or ordered for diabetes screening at the visits $(n=8375)$.

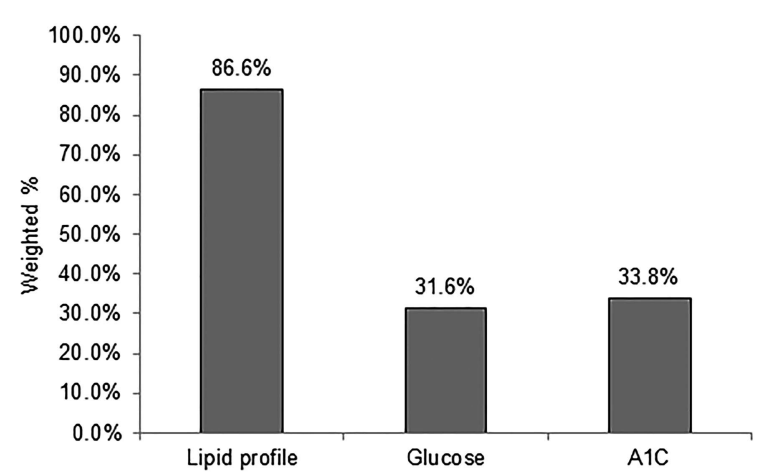


Figure 4. Treatment provided during visits with prediabetes $(n=5406)$.

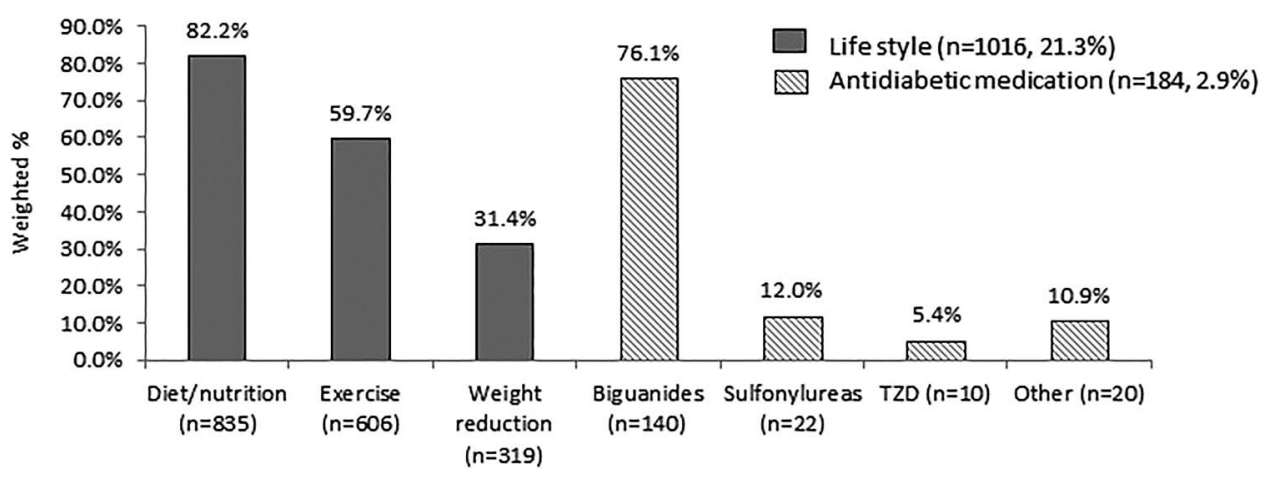

tion is more likely to have Medicare, and coverage of certain screening tests is lacking. Medicare will cover screening for diabetes by using fasting glucose or a glucose tolerance test. ${ }^{19}$ Even though the $\mathrm{ADA}$ recommends the $\mathrm{A} 1 \mathrm{C}$ test as a viable method for screening, Medicare will only cover an A1C for patients already diagnosed with DM. It has been suggested that coverage of this test may increase DM screening in this patient population due to physician preference. ${ }^{18}$ This study also shows a slight preference for A1C (33.8\%) over fasting glucose $(31.6 \%)$, as more visits involved the use of $\mathrm{A} 1 \mathrm{C}$; however, there is no significant difference. This may also account for why patients with private insurance were more likely to be screened compared to patients with Medicare, Medicaid, other, or uninsured.

Other factors associated with screening include the number of chronic conditions, race, and BMI. The more chronic conditions a patient presented with, the more likely they would be to receive screening. In addition, patients with higher BMI and those who were nonwhite were more likely receive screening for DM, which is similar to previous studies using patient self-reported data and national epidemiology data regarding the incidence and prevalence of DM. ${ }^{6,16,17}$ Increased BMI and nonwhite race or ethnicity can increase the risk of developing DM individually, so these results indicate that guideline recommendations are being used appropriately in these patients. ${ }^{1}$ There was not an association with geographic location in regard to receiving screening or not, which is somewhat surprising considering the prevalence of DM is higher in the South. ${ }^{6}$ It is noted that patients with prediabetes were twice as likely to receive annual screening compared to those without prediabetes, which is appropriate.
The USPSTF's recommendations provide a brief review of the benefits and harms of early detection. ${ }^{4}$ In the current recommendation, published in 2015, there was insufficient direct evidence to support early detection leads to significant improvements in mortality or CVD, and the harms of early detection are attributed to short-term anxiety over laboratory testing. An update to screening recommendations for DM is in progress and includes conducting a systematic review for updated evidence related to benefits and harms of early detection. ${ }^{20}$

The second objective of this study was to determine what interventions, if any, were given or recommended to patients who were diagnosed with prediabetes. Approximately $5 \%$ of the visits analyzed included a diagnosis of or previous laboratory testing indicative of prediabetes. These results are lower than the $11.6 \%$ of US adults that reported being aware of having prediabetes. ${ }^{6}$ Of these visits, only $21 \%$ of patients received lifestyle as a treatment modality, whereas less than $3 \%$ received pharmacologic therapy. These results are similar to a previous study using data from the NAMCS database, which identified $23 \%$ of patients were given lifestyle or pharmacologic treatment for diagnosed or undiagnosed prediabetes in $2012 .^{21}$ This demonstrates that more than $75 \%$ of patients identified as being at risk for developing DM in the future were not provided evidence-based recommendations, including pharmacologic or lifestyle modifications, to delay or prevent the disease. The upward trend, albeit small, of screening patients at risk of developing DM may be promising as more focus is being placed on prevention. However, the lack of treatment provision, especially lifestyle management, is disappointing.

Intense lifestyle interventions, such as the DPP, can reduce the risk of developing DM by more than 
$50 \%$ up to 3 years after participation and by approximately $30 \%$ up to 10 years after participation. ${ }^{22}$ In addition, lifestyle interventions have been more effective in patients greater than 60 years of age at least 10 years after participation, according to follow up data from the Diabetes Prevention Program Outcome Study. The USPSTF's review found sufficient evidence that intensive lifestyle interventions lower cardiovascular risk in patients who are overweight or obese with additional risk factors and that the harms of such treatment were minimal. $^{4}$

Perhaps, though, future studies will demonstrate a higher percentage of patients treated for prediabetes as coverage of effective preventive services increases, particularly in older adults. Medicare has extended coverage to the DPP for adults aged 65 and older with prediabetes to be effective in $2018 .^{6}$ The CDC provides economic estimates for states, employers, and insurers that are considering implementing the DPP at their website, https://nccd.cdc. gov/Toolkit/DiabetesImpact/. Prevention of DM by using evidence-based, cost-effective options, such as the DPP or pharmacologic therapy like metformin, can lead to decreased overall medical costs by avoiding or delaying the treatment of DM. $^{23}$

The strengths to this study include the generalizability to the US population and the amount of data available to analyze in the NAMCS database. However, several limitations are present. The NAMCS contains cross-sectional data through 2015 and does not link longitudinal individual patient visits together. The more recent emphasis on preventive services particularly for DM may not be reflected in the data available. In addition, NAMCS reflected a snapshot of the diabetes screening provision at the visits. First, it is not possible to determine true adherence to the screening recommendations due to cross-sectional study design. For instance, patients with normal screening results may appropriately be screened once every 3 years rather than annually for those with abnormal screening results. However, it is only possible to determine whether screening had occurred at the visits reported by NAMCS. Second, the NAMCS did not provide further details to differentiate whether the screening test was provided, ordered, or referred at the visit. An assumption was made in this study. All screening tests were provided at the visit if the answers to this question were yes. The actual screening provision could be overestimated. Third, TG and HDL lab test results were collected from 2012 to 2014. The visits based on this criterion could be understated in 2015. In addition, a random plasma glucose $\geq \mathrm{x} 200 \mathrm{mg} / \mathrm{dL}$ was not included as a criterion to identify patients with prediabetes. This omission could result in a selection bias in the study. Determining the appropriate use of pharmacologic therapy was outside the scope of the study, so more research is needed in this area. Finally, the contents of the lifestyle management were not available in the NAMCS data. Thus, the quality of the health education to treat prediabetes could not be assessed.

\section{Conclusion}

More attention is needed to improve the screening rate of adults with risk factors for developing DM, in addition to the use of interventions to prevent or delay the development of DM in patients with prediabetes. The use of available resources to incorporate screening into practice and to engage patients in education and lifestyle interventions may be helpful.

To see this article online, please go to: http://jabfm.org/content/ 32/2/209.full.

\section{References}

1. American Diabetes Association. Classification and diagnosis of diabetes: Standards of Med Care in Diabetes-2018. Diabetes Care. 2018;41(Supplement 1):S13-S27.

2. Handelsman Y, Bloomgarden ZT, Grunberger G, et al. American Association of Clinical Endocrinologists and American College of Endocrinology-clinical practice guidelines for developing a diabetes mellitus comprehensive care plan-2015. Endocr Pract. 2015;21:413-37.

3. Pippitt K, Li M, Gurgle H. Diabetes mellitus: screening and diagnosis. Am Fam Physician 2016;93: 103-9.

4. Siu AL, on behalf of the USPSTF. Screening for abnormal blood glucose and type 2 diabetes mellitus: U.S. Preventive Services Task Force recommendation statement. Ann Intern Med. 2015;163:861-8.

5. Centers for Disease Control and Prevention. Diabetes Report Card 2014. Available from: https://www.cdc. gov/diabetes/pdfs/library/diabetesreportcard2014.pdf. Published 2015. Accessed December 3, 2018.

6. Centers for Disease Control and Prevention. Diabetes Report Card 2017. Available from: https://www.cdc. gov/diabetes/pdfs/library/diabetesreportcard2017-508. pdf. Published 2018. Accessed December 3, 2018. 
7. Centers for Disease Control and Prevention. National Diabetes Statistics Report, 2017. Available from: https://www.cdc.gov/diabetes/pdfs/data/statistics/national-diabetes-statistics-report.pdf. Accessed December 3, 2018. Published 2017.

8. Ligthart S, van Herpt T, Leening M, et al. Lifetime risk of developing impaired glucose metabolism and eventual progression from prediabetes to type $2 \mathrm{di}-$ abetes: a prospective cohort study. Lancet Diabetes Endocrinol. 2016;4:44-51.

9. Tabak A, Herder C, Rathmann W, Brunner E, Kivimaki $M$. Prediabetes: a high-risk state for diabetes development. Lancet. 2012;379:2279-90.

10. American Diabetes Association. Economic costs of diabetes in the U.S. in 2017. Diabetes Care. 2018; 41:917-28.

11. American Medical Association and Centers for Disease Control and Prevention. Preventing type 2 diabetes: a guide to refer your patients with prediabetes to an evidence-based diabetes prevention program. Available from: https://preventdiabetesstat. org/toolkit.html. Accessed December 3, 2018.

12. American Diabetes Association. Prevention or delay of type 2 diabetes: Standards of Med Care in Diabetes-2018. Diabetes Care. 2018;41(Suppl 1):S51-S4.

13. National Center for Health Statistics. Ambulatory health care data. Atlanta, GA: Centers for Disease Control and Prevention [updated 2018 Nov 7; cited 2018 Nov 14]. Available from: https://www.cdc.gov/ nchs/ahcd/index.htm. Accessed November 14, 2018.

14. Goodman RA, Posner SF, Huang ES, Parekh AK, Koh HK. Defining and measuring chronic conditions: imperatives for research, policy, program, and practice. Prev Chronic Dis. 2013;10:E66.

15. National Center for Health Statistics. 2015 NAMCS Micro-data file documentation. [updated 2017 Sep 11; cited 2018 Nov 14]; Available from: ftp://ftp.cdc.gov/ pub/Health_Statistics/NCHS/Dataset_Documentation/ NAMCS/doc2015.pdf. Accessed November 15, 2018.
16. Kiefer MM, Silverman JB, Young BA, Nelson KM. National patterns in diabetes screening: data from the National Health and Nutrition Examination Survey (NHANES) 2005-2012. J Gen Intern Med 2015;30:612-8.

17. Bullard KM, Ali MK, Imperatore G, et al. Receipt of glucose testing and performance of two US diabetes screening guidelines, 2007-2012. PLoS One. 2015; 10:e0125249.

18. Mehta S, Mocarski M, Wisniewski T, Gillespie K, Narayan KMV, Lang K. Primary care physicians' utilization of type 2 diabetes screening guidelines and referrals to behavioral interventions: a surveylinked retrospective study. BMJ Open Diabetes Res Care. 2017;5(1).e000406.

19. American Hospital Association. Reminder: Medicare provides coverage of diabetes screening tests. Available from: https://www.cms.gov/Outreach-and-Education/ Medicare-Learning-Network-MLN/MLNMatters Articles/downloads/SE0821.pdf. Published 2018. Accessed July 6, 2018.

20. U.S. Preventive Services Task Force. Final research plan: abnormal blood glucose and type 2 diabetes mellitus: screening. 2018; Available from: https://www.uspreventiveservicestaskforce.org/Page/Document/finalresearch-plan/abnormal-blood-glucose-and-type-2diabetes-mellitus-screening. Published 2018. Accessed December 3, 2018.

21. Mainous AG, Tanner RJ, Baker R. Prediabetes diagnosis and treatment in primary care. J Am Board Fam Med 2016;29:283-5.

22. Diabetes Prevention Program Research Group. 10year follow-up of diabetes incidence and weight loss in the Diabetes Prevention Program Outcomes Study. Lancet. 2009;374:1677-86.

23. Diabetes Prevention Program Research Group. The 10-Year cost-effectiveness of lifestyle intervention or metformin for diabetes prevention. Diabetes Care. 2012;35:723-30. 